\title{
Prognostic value of the pretreatment neutrophil-to-lymphocyte ratio in patients with advanced gastrointestinal stromal tumors treated with sunitinib after imatinib failure
}

\author{
PAWEŁ SOBCZUK ${ }^{1,2^{*}}$, PAWEŁ TETERYCZ ${ }^{1 *}$, IWONA LUGOWSKA ${ }^{1,3,4}$, ANNA KLIMCZAK $^{1}$, \\ ELŻBIETA BYLINA $^{1,4,5}$, ANNA M. CZARNECKA ${ }^{1,6}$, HANNA KOSELA-PATERCZYK $^{1}$, \\ CZESŁAW OSUCH $^{7}$, JOANNA STREB ${ }^{7}$ and PIOTR RUTKOWSKI ${ }^{1}$
}

\begin{abstract}
${ }^{1}$ Department of Soft Tissue/Bone Sarcoma and Melanoma, Maria Sklodowska-Curie Institute-Oncology Center, 02-781 Warsaw;
${ }^{2}$ Department of Experimental and Clinical Physiology, Laboratory of Centre for Preclinical Research, Medical University of Warsaw, 02-097 Warsaw; ${ }^{3}$ Department of Biostatistics, Institute of Mother and Child, 01-211 Warsaw; ${ }^{4}$ Early Phase Clinical Trial Unit; ${ }^{5}$ Clinical Trial Administrative Unit, Maria Sklodowska-Curie Institute-Oncology Center, 02-781 Warsaw;

${ }^{6}$ Department of Experimental Pharmacology, Mossakowski Medical Research Centre, Polish Academy of Sciences, 02-106 Warsaw; ${ }^{7}$ Department of Oncology, Iagiellonian University, 31-531 Cracow, Poland
\end{abstract}

Received March 27, 2019; Accepted July 8, 2019

DOI: $10.3892 / 01.2019 .10622$

\begin{abstract}
The neutrophil-to lymphocyte ratio (NLR) has been proven to be correlated with outcomes in various cancer types, including gastrointestinal stromal tumors (GIST). There is limited data regarding the clinical value of NLR during second line therapy after failure of imatinib and there is an urgent need for more precise predictive factors for therapy. The aim of this study was to assess the association of the pretreatment NLR with progression free survival (PFS) and overall survival (OS) in patients with unresectable/metastatic GIST treated with sunitinib in a second line of treatment. In this analysis 146 out of 230 patients with unresectable/metastatic GIST were included, who were treated between 2005 and 2016 with sunitinib after failure of imatinib, with complete clinical data. In all patients, the NLR was assessed at baseline. The NLR cutoff of 2.4 was selected. The Kaplan-Meier method with the long-rank test and Cox proportional hazards model were applied for statistical analysis. Median PFS was 12.4 months with a 2-year rate of $27.1 \%$ and a 5 -year rate of $4.8 \%$. Median OS was 22.8 months, whereas 2 - and 5-year rates were 47.8 and $13.8 \%$, respectively. Patients with NLR $>2.4$ had significantly
\end{abstract}

Correspondence to: Professor Piotr Rutkowski, Department of Soft Tissue/Bone Sarcoma and Melanoma, Maria Sklodowska-Curie Institute-Oncology Center, Wilhelma Konrada Roentgena 5, 02-781 Warsaw, Poland

E-mail: piotr.rutkowski@coi.pl

*Contributed equally

Key words: gastrointestinal stromal tumor, sunitinib, neutrophilto-lymphocyte ratio, prognostic factors, KIT shorter OS: Median OS was 30 months for NLR $\leq 2.4$ vs. 16.4 months for NLR $>2.4(\mathrm{P}=0.002)$; median PFS was 18.2 vs. $9.6(\mathrm{P}=0.075)$, respectively. In a multivariate model adjusted for mitotic index, primary location of tumor and driver mutation in KIT exon 11, NLR was proven to be independently associated with OS (HR 1.92, 95\% CI 1.27-2.9, $\mathrm{P}=0.002$ ) but not PFS (HR 1.31, 95\% CI 0.89-1.93, $\mathrm{P}=0.17$ ). The present data demonstrate that NLR can serve as an independent prognostic factor for patients with advanced GIST treated with sunitinib.

\section{Introduction}

Gastrointestinal stromal tumor (GIST) is a rare neoplasm with an unadjusted incidence estimated at around 1 in 100,000 per year, however it is the most common mesenchymal tumor of the gastrointestinal tract (1). GISTs arise mainly in the stomach and small intestine, but they can be found anywhere in the gastrointestinal tract (2). Several genetic alterations are involved in the pathogenesis of GISTs with the two most common being-activating mutations of $K I T$ gene and platelet-derived growth factor receptor- $\alpha$ (PDGFRA) gene mutations $(3,4)$. Surgery remains the gold standard and only curative option in the treatment of localized disease, but management of advanced stage tumors is very challenging. Significant improvement in the treatment of advanced GIST has been achieved due to better understanding of the molecular background and introduction of tyrosine kinase inhibitors (TKIs). The first TKI that revolutionized management of GIST was imatinib-a small molecule selective inhibitor of KIT, PDGFRA and BCR-ABL fusion protein. Imatinib is currently the standard of care in the first-line treatment in advanced (metastatic and/or inoperable) disease and the standard adjuvant therapy after resection of primary tumors at high risk of relapse (5). Despite its spectacular improvements to patient survival, response to imatinib therapy is limited by time and the majority of patients develop resistance to this 
TKI. Between 10 and $15 \%$ of GISTs are primary resistant to imatinib, whereas during the first 2 years from start of imatinib, approximately $50 \%$ develop secondary resistance (6). In the case of progression, the dose of imatinib can be increased if it has not been used previously at maximal dosage, or the patient can be switched to other drugs.

Sunitinib is the only approved drug for the second-line treatment of GISTs $(5,7)$, It is an oral multitargeted receptor tyrosine kinase inhibitor, that targets stem cell factor receptor KIT, PDGFRA/B, FMS-like tyrosine kinase-3 receptor (FLT3), the vascular endothelial growth factor receptors (VEGFR-1, VEGFR-2, VEGFR-3), and the glial cell-line derived neurotrophic factor receptor (RET). Results from clinical trials have shown significant efficacy of sunitinib in imatinib-resistant patients with a median progression free survival (PFS) of 6-8 months compared to a median PFS of 1-2 months on placebo (7).

Prognostic and predictive factors are investigated to identify the patients who benefit the most from sunitinib therapy. The prognostic value of blood morphology-derived factors was confirmed to correlate with survival in several cancers, including GIST. Neutrophil-to lymphocyte ratio (NLR) was shown to play a prognostic role in primary GIST after radical resection, in patients receiving imatinib as adjuvant therapy or in the first-line of treatment for advanced disease. However, the NLR has not been evaluated in patients treated with sunitinib in the second-line settings.

The important factor associated with patient survival and response to treatment of GIST is the genetic profile of the tumor. Primary tumor genotype was found to be associated with PFS and OS in patients treated with imatinib. Patients with KIT exon 11 mutations show greater benefit from imatinib therapy than patients with other mutations (8), whereas exon 18 PDGFRA D842V mutant GIST are not sensitive to imatinib and other tyrosine kinase inhibitors (9). During treatment with sunitinib, better outcomes were observed in patients harboring primary mutations in KIT exon 9 than in exon $11(10,11)$.

The aim of this study was to evaluate the prognostic and predictive value of NLR in patients with advanced GIST treated with sunitinib as a second line treatment after imatinib failure. Additionally, we have investigated the impact of the baseline tumor genotype on patient survival.

\section{Materials and methods}

Patients. Between September 1, 2005 and June 30, 2016, 232 patients with histologically confirmed unresectable and/or metastatic CD117-positive GIST were treated with sunitinib as a second-line treatment at a reference sarcoma center, Department of Soft Tissue/Bone Sarcoma and Melanoma, Maria Sklodowska-Curie Institute-Oncology Center in Warsaw, Poland. Of these, 146 patients that had complete laboratory test results were included in this retrospective study. Data were extracted from institutional medical records with the ONKOSYS Medstreamer software. The local Bio-Ethics Committee has approved the study, according to the guidelines on good clinical practice and local bylaws. At the beginning of treatment, each patient provided informed consent for use of their data for future studies. Collected data included the following clinicopathologic characteristics: Age, gender, tumor size, tumor location, number of mitosis, mutational status and laboratory findings, PFS, and overall survival.

NLR was calculated as the ratio of absolute neutrophil count to absolute lymphocyte count obtained from the complete blood count. To evaluate prognostic impact of the initial status, the NLR was evaluated at baseline. Further assessments were carried out after 3 months of treatment and upon progression or at last observation.

Genotype status before TKI therapy for the presence of mutations in the KIT (exons 9,11,13,17) and PDGFRA (exons $12,14,18$ ) genes, including PDGFRA 18 D842V mutation, was available for $72(49.3 \%)$ cases.

All patients were treated with imatinib in the first line and had objective disease progression diagnosed by computed tomography (CT) scans before starting sunitinib treatment. Sunitinib was prescribed as part of a standard scheme of therapy. Patients received sunitinib orally at a starting dose of $50 \mathrm{mg}$ once daily in 6-week cycles: 4 weeks of treatment and 2 weeks without. According to the standard guidelines, dose reduction (to $37.5 \mathrm{mg}$ or $25 \mathrm{mg}$ ), treatment interruption or modulation to dosage of $37.5 \mathrm{mg}$ on a continuous schedule could be used to manage adverse events and optimize the benefit-risk profile. Treatment was stopped when disease progression was observed on scans, when the patient presented with signs of clinical progression, when unacceptable adverse events occurred, or in the case of death. Patient follow-up consisted of regular physical examinations and laboratory assessment every 4-6 weeks, and serial CT scans performed every 2-3 month according to the strict setting of the drug program reimbursed by national health insurance in our country. The evaluation of tumor response was carried out based on the Response Evaluation Criteria in Solid Tumors (RECIST) version 1.1 (12). In case of progression, patients were treated with other TKI, or they received the best supportive care only. If feasible, they were included in clinical trials with new compounds.

Statistical analysis. All statistical analyses were performed using the $\mathrm{R}$ language environment version 3.5.2. The Kaplan-Meier method with the log-rank tests for bivariate comparisons was used for calculation of survival. The aim of this study was to assess the correlation of NLR value before treatment and at progression or last follow-up with patient PFS and OS times in advanced GIST cases treated with sunitinib in the second line. Overall survival was defined as the time from initiation of sunitinib treatment until the most recent follow-up or death of any cause. The Polish National Death Registry was used to confirm the dates of all patient deaths. PFS was defined as the time from initiation of sunitinib treatment until disease progression, death of any cause, or most recent follow-up. The following variables were included univariate and multivariate analyses of factors influencing survival: demographic data (sex, age at the start of sunitinib therapy $<55$ or $\geq 55$ years-a cutoff value of 55 was selected because it is the most robust cut off closest to the median value of 57), primary tumor genotype (KIT exon 11 mutations, KIT exon 9 mutations, PDGFRA 18 $D 842 \mathrm{~V}$ mutation, other KIT mutations, other PDGFRA mutations, wild-type), the maximal diameter of the largest tumor, primary tumor location (gastric vs non-gastric), the mitotic index of the tumor $[\leq 5 / 50$ high power field on microscopy (HPF) vs. $>5 / 50 \mathrm{HPF}]$ and baseline (1-7 days before start of 
Table I. Distribution of clinicopathological characteristics of patients in overall population and stratified by pre-treatment NLR.

\begin{tabular}{|c|c|c|c|c|}
\hline Characteristics & Overall patients $\mathrm{n}(\%)$ & $\mathrm{NLR} \leq 2.4 \mathrm{n}(\%)$ & NLR>2.4 n (\%) & P-value \\
\hline \multicolumn{5}{|l|}{ Sex } \\
\hline Male & $81(55.5)$ & $36(24.7)$ & $45(30.8)$ & \multirow[t]{2}{*}{0.335} \\
\hline Female & $65(44.5)$ & $35(24.0)$ & $30(20.5)$ & \\
\hline \multicolumn{5}{|l|}{ Age, y } \\
\hline$<55$ & $58(39.7)$ & $42(28.8)$ & $46(31.5)$ & \multirow[t]{2}{*}{0.921} \\
\hline$\geq 55$ & $88(60.2)$ & $29(19.9)$ & $29(19.9)$ & \\
\hline \multicolumn{5}{|l|}{ Tumor location } \\
\hline Gastric & $36(24.7)$ & $21(14.7)$ & $15(10.4)$ & \multirow[t]{2}{*}{0.312} \\
\hline Nongastric & $107(73.4)$ & $50(35.0)$ & $57(39.9)$ & \\
\hline \multicolumn{5}{|l|}{ Tumor size, $\mathrm{cm}$} \\
\hline$<10 \mathrm{~cm}$ & $43(29.5)$ & $21(14.4)$ & $22(15.1)$ & \multirow[t]{3}{*}{0.447} \\
\hline$>10 \mathrm{~cm}$ & $72(49.3)$ & 32 & $40(27.4)$ & \\
\hline Unknown & $31(21.2)$ & $18(12.3)$ & $13(8.9)$ & \\
\hline \multicolumn{5}{|l|}{ Mitotic index } \\
\hline$<5 / \mathrm{HPF}$ & $24(16.4)$ & $9(6.1)$ & $15(10.2)$ & \multirow[t]{3}{*}{0.485} \\
\hline$\geq 5 / \mathrm{HPF}$ & $70(48.0)$ & $36(24.7)$ & $34(23.3)$ & \\
\hline Unknown & $52(35.6)$ & $26(17.8)$ & $26(17.8)$ & \\
\hline \multicolumn{5}{|l|}{ Pre-treatment NLR } \\
\hline$<2.4$ & $71(48.6)$ & NA & NA & \\
\hline$\geq 2.4$ & $75(51.4)$ & NA & NA & \\
\hline \multicolumn{5}{|l|}{ Mutational status } \\
\hline KIT 11 & $43(29.5)$ & 21 & 22 & \multirow[t]{7}{*}{0.999} \\
\hline$K I T 9$ & $15(10.3)$ & 8 & 7 & \\
\hline PDGFRA $18 \mathrm{D} 842 \mathrm{~V}$ & $4(2.7)$ & 1 & 3 & \\
\hline Other PDGFRA & $0(0)$ & 0 & 0 & \\
\hline Other $K I T$ & $1(0.7)$ & $1(0.7)$ & 0 & \\
\hline Wild-type & $9(6.2)$ & $4(2.7)$ & $5(3.4)$ & \\
\hline Unknown & $74(50,7)$ & 36 & 38 & \\
\hline
\end{tabular}

HPF, high-power field; NLR, neutrophil-to-lymphocyte ratio.

sunitinib therapy) NLR ( $\leq 2.4$ vs. $>2.4)$. All covariates significant at the $20 \%$ level in the univariate model were included in a multivariate analysis, which was performed with Cox proportional hazards models, applying the stepwise model building procedure. $\mathrm{P}<0.05$ was considered to indicate a statistically significant difference.

An NLR cutoff of 2.4 was selected based on maximally selected log-rank statistics implemented in the maxstat package with inclusion of recent literature data. Based on the statistical calculations a cutoff value of 2.4 resulted in the best diversification of the population into subpopulations with varying degrees of positive or negative prognosis.

\section{Results}

Patient characteristics. One hundred forty-six patients (81 males and 65 females) were included in the study. The median age at the start of sunitinib therapy was 74 years (range 18-84 years). Thirty-six (24.7\%) patients had tumors located in the stomach, whereas 107 (73.3\%) had tumors presenting in non-gastric locations. There were no significant differences between the NLR $\leq 2.4$ and NLR $>2.4$ groups. The clinicopathological characteristics of the study population are presented in Table I.

In the group of 72 patients with available tumor genotype data, $59.7 \%(n=43)$ of GIST cases exhibited KIT exon 11 mutations, $20.8 \%(\mathrm{n}=15)$ exhibited $K I T$ exon 9 mutations, $5.5 \%$ (n=5) PDGFRA 18 D842V mutation, 1 patient had other KIT mutations, and $12.5 \%(n=9)$ of tumors were of the wild-type with no mutations detected.

The median NLR at baseline was 2.47 (range 0.6-27.6, mean 3.4 \pm 3.5 ) whereas the median NLR at progression (or last available follow-up) was 1.87 (range, 0.3-23.3, mean 2.6 \pm 2.8 ). Seventy-one patients (48.6\%) had a baseline NLR $\leq 2.4$ and 75 patients $(51.4 \%)$ had a baseline NLR $>2.4$.

Sunitinib treatment outcomes. The median follow-up time was 70.1 months (range: 64-104.5). One hundred and fifteen patients $(78.8 \%)$ progressed during sunitinib therapy. At the time of the analysis 28 patients $(26 \%)$ were alive. Eight patients 

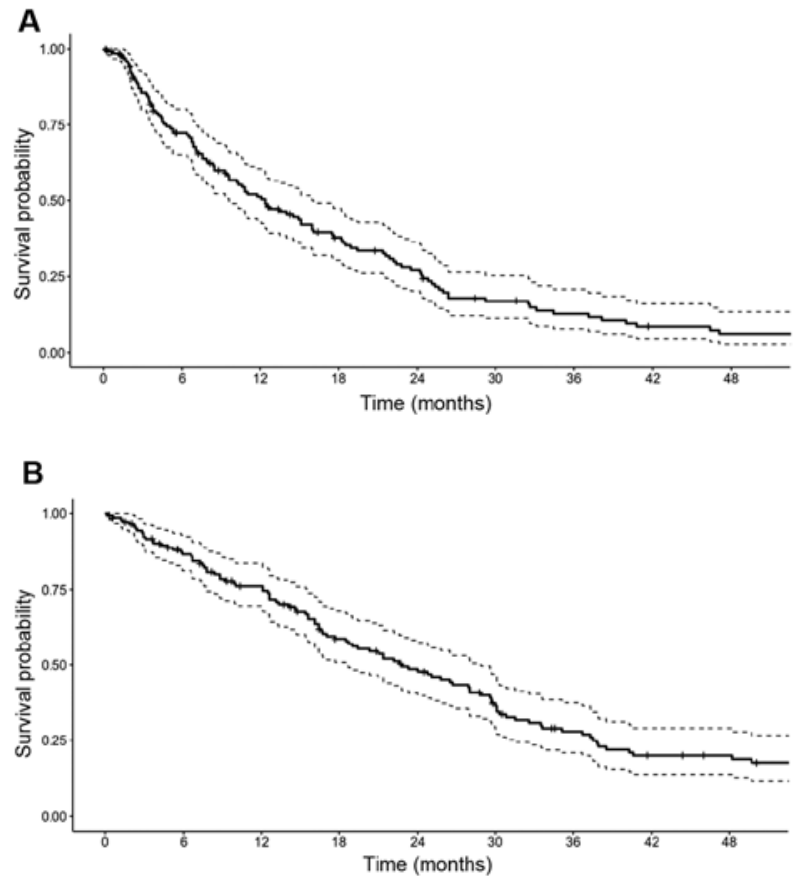

Figure 1. Kaplan-Meier curves showing (A) progression-free survival and (B) overall survival in patients treated with sunitinib.

were lost during follow-up. The median PFS on sunitinib treatment was 12.4 months (95\% CI 9.6-16), the 2-year rate was $27.1 \%$ and the 5 -year rate was $4.8 \%$ (Fig. 1A). The median OS was 22.8 months (95\% CI 18.5-28.9), 2-year rate was $47.8 \%$ and 5 -year rate $13.8 \%$ (Fig. 1B).

The median PFS in patients with a baseline NLR $\leq 2.4$ and NLR $>2.4$ were 18.2 and 9.6 months $(\mathrm{P}=0.075$; Fig. $2 \mathrm{~A})$ respectively, whereas the median OS were 30 and 16.4 months $(\mathrm{P}=0.002$; Fig. 2B), respectively.

Univariate analysis of factors associated with PFS and OS. Male sex (HR 1.65, 95\% CI 1.13-2.4, $\mathrm{P}=0.009$ ) and mutations in $K I T$ exon 11 (HR 2.23, 95\% CI 1.29-3.84, $\mathrm{P}=0.004$ ) were significantly associated with PFS in the univariate analysis (Table II). NLR was significantly associated with PFS when treated as a continuous variable (HR 1.06 per unit change, 95\% CI: 1.001-1.123, $\mathrm{p}=0.045)$. Male sex (HR 1.55, 95\% CI $1.05-2.28, \mathrm{p}=0.026$ ), age $\geq 55$ (HR $1.65,95 \%$ CI $1.1-2.48$, $\mathrm{P}=0.015)$, mutations in $K I T$ exon 11 (HR 2.64, 95\% CI 1.47-4.73, $\mathrm{P}=0.001$ ), unknown mutational status (HR 2.02, 95\%CI 1.15-3.54), and baseline NLR >2.4 (HR 1.85, 95\% CI $1.26-2.72, \mathrm{P}=0.002)$, were significantly associated with shorter OS in the univariate analysis (Table III).

Multivariate analysis of factors associated with PFS and $O S$. The multivariate analysis revealed that only mutations in $K I T$ exon 11 (HR 2.38, 95\% CI 1.27-4.47, $\mathrm{P}=0.007$ ) were associated with shorter PFS on sunitinib (Table II). NLR $>2.4$ was not associated with PFS in the multivariate analysis (HR 1.31, 95\%CI 0.89-1.93, $\mathrm{P}=0.17$ ). Mutations in KIT exon 11 (HR 3.39, 95\% CI 1.73-6.63, $\mathrm{P}<0.001$ ), unknown mutational status (HR 2.29, 95\% CI 1.19-4.39), age $\geq 55$ (HR $1.63,95 \%$ CI $1.06-2.53, \mathrm{P}=0.028)$ and baseline NLR $>2.4$ (HR $1.92,95 \%$ CI $1.27-2.9, \mathrm{P}=0.002$ ), were independently
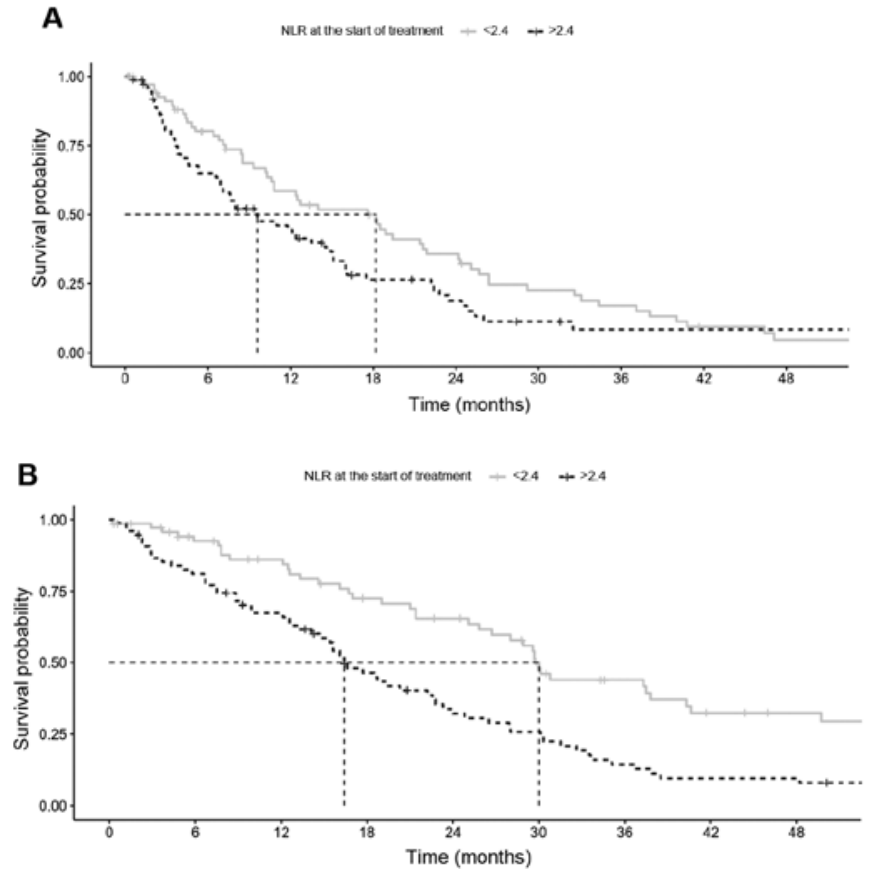

Figure 2. Kaplan-Meier curves showing (A) progression-free survival and (B) overall survival in patients treated with sunitinib, stratified by the pre-treatment neutrophil-to-lymphocyte ratio.

associated with shorter OS in the multivariate analysis (Table III).

\section{Discussion}

The prognostic value of NLR was previously shown for a variety of malignancies, including soft tissue sarcomas (13), colorectal, renal, lung and pancreatic cancers (14-16). There is a growing body of data supporting the usefulness of NLR in prognostication in GISTs. Its role as an independent prognostic factor of RFS in localized primary GISTs has already been presented (17-19). Moreover, in this group of patients increased NLR was correlated with shorter OS $(18,20)$. However, some authors present opposite results (21). High NLR was also associated with the characteristic features of high-risk tumors, what suggests that high risk GISTs can promote systemic inflammation (20). Much less is known about the role of NLR in patients treated systemically due to metastatic/unresectable GIST. In our previous study we reported that NLR $>2.7$ at baseline was significantly associated with poor OS and PFS in patients receiving imatinib in the first line of treatment of advanced GIST (10). To our knowledge, this is the first study showing that pretreatment NLR may be associated with OS in advanced GIST treated with sunitinib as a second-line therapy. In this study, we have found that baseline NLR $>2.4$ is a negative independent prognostic factor for OS.

Direct mechanisms that clarify the poor survival outcomes in patients with high blood NLR are poorly understood. One potential explanation of this phenomenon could be connection between high NLR and the systemic inflammation induced by tumor cells and associated host cells. There is increasing evidence of an association between cancer and inflammation; in fact, inflammation is now considered a hallmark of 
Table II. Univariate and multivariate analysis of association of clinicopathological factors with progression-free survival.

\begin{tabular}{|c|c|c|c|c|c|c|}
\hline \multirow[b]{2}{*}{ Variable } & \multicolumn{3}{|c|}{ Univariate analysis } & \multicolumn{3}{|c|}{ Multivariate analysis } \\
\hline & HR & $95 \% \mathrm{CI}$ & P-value & HR & $95 \% \mathrm{CI}$ & P-value \\
\hline \multicolumn{7}{|l|}{ Sex } \\
\hline Female & 1 & & & 1 & & \\
\hline Male & 1.65 & $1.13-2.4$ & 0.009 & 1.35 & $0.9-2.03$ & 0.15 \\
\hline \multicolumn{7}{|l|}{ Age } \\
\hline$<55$ & 1 & & & 1 & & \\
\hline$\geq 55$ & 1.23 & $0.84-1.8$ & 0.299 & 1.26 & $0.84-1.9$ & 0.27 \\
\hline \multicolumn{7}{|c|}{ Tumor location } \\
\hline Nongastric & 1 & & & 1 & & \\
\hline Gastric & 1.32 & $0.84-2.07$ & 0.23 & 1.29 & $0.78-2.11$ & 0.32 \\
\hline \multicolumn{7}{|l|}{ Tumor size } \\
\hline 1 & 1 & & & 1 & & \\
\hline 2 & 1.33 & $0.87-2.05$ & 0.19 & 1.25 & $0.80-1.96$ & 0.32 \\
\hline Unknown & 1.24 & $0.7-2.15$ & 0.45 & 1.16 & $0.57-2.38$ & 0.68 \\
\hline \multicolumn{7}{|c|}{ Mitotic index } \\
\hline$<5 / \mathrm{HPF}$ & 1 & & & 1 & & \\
\hline$\geq 5 / \mathrm{HPF}$ & 1.16 & $0.69-1.97$ & 0.58 & 0.97 & $0.56-1.69$ & 0.92 \\
\hline Unknown & 1.22 & $0.7-2.15$ & 0.48 & 1.02 & $0.5-2.07$ & 0.96 \\
\hline \multicolumn{7}{|c|}{ Pre-treatment NLR } \\
\hline$\leq 2.4$ & 1 & & & 1 & & \\
\hline$>2.4$ & 1.4 & $0.97-2.03$ & 0.076 & 1.31 & $0.89-1.93$ & 0.17 \\
\hline \multicolumn{7}{|c|}{ Mutational status } \\
\hline KIT 11 & 2.23 & $1.29-3.84$ & 0.004 & 2.38 & $1.27-4.47$ & 0.007 \\
\hline Other & 1 & & & 1 & & \\
\hline Unknown & 1.58 & $0.95-2.63$ & 0.08 & 1.64 & $0.9-2.97$ & 0.11 \\
\hline
\end{tabular}

CI, confidence interval; HPF, high-power field; HR, hazard ratio; NLR, neutrophil-to-lymphocyte ratio.

cancer (22). Moreover, the tumor niche and tumor-associated inflammation play crucial roles in carcinogenesis, progression, and the development of metastasis. Inflammatory processes in the tumor are reflected by changes in leucocyte blood count or increased levels of circulating cytokines (23). Perez et al (19) suggested that NLR in GIST is mainly determined by the absolute neutrophil count because there is no established link between blood lymphocytes and prognosis in solid tumors. Rather, the lymphocyte count alone can be considered a measure of patient-specific immune response against cancer with limited prognostic value. Neutrophilia can inhibit the immune system by suppressing the cytolytic activity of lymphocytes, natural killer cells, or activated T cells $(24,25)$. High NLR was found to be related to an increase in macrophage infiltration of peritumoral tissue and elevated systemic concentrations of IL-17, (26), IL-12, IL-8, IL-7, IL-6, IL-1ra, MCP-1 and PDGFBB (27). Moreover, neutrophils can secrete tumor growth promoting factors, including HGF, VEGF, IL-8, IL-6, MMPs and elastases and thus lead to the formation of tumor stimulating microenvironment, proliferation, migration, and invasiveness of tumor cells (25). Elevated NLR may indicate elevated concentrations of circulating cytokines, including some factors promoting tumor growth such as transforming growth factor-beta (TGF-beta) (28). Moreover, TGF-beta can promote an increase in the number, activation, and survival of neutrophils and reduce the number of lymphocytes (29). Altogether, NLR and circulating inflammatory cytokines can perpetuate a tumor microenvironment and reflect aggressive behavior.

Despite an almost 9-month difference in PFS between patients with NLR $\leq 2.4$ and NLR $>2.4$, we have not found NLR to be an independent predictive factor for PFS, what was also shown in the population of patients receiving imatinib in the first-line (10). The lack of a statistical correlation between NLR and PFS in this study may have resulted from the study's small sample size.

In addition to NLR, we found that primary mutations in exon 11 KIT were associated with worse PFS and OS in patients treated with sunitinib in the second line, with a 2.4- and 3.4-fold increase in hazard ratios for PFS and OS, respectively. This finding is in alignment with our previous observation where we have reported that tumors initially bearing KIT exon 9 mutations and wild-type tumors had significantly higher 1-year PFS rates than those carrying KIT 
Table III. Univariate and multivariate analysis of association of clinicopathological factors with progression-free survival.

\begin{tabular}{|c|c|c|c|c|c|c|}
\hline \multirow[b]{2}{*}{ Variable } & \multicolumn{3}{|c|}{ Univariate analysis } & \multicolumn{3}{|c|}{ Multivariate analysis } \\
\hline & HR & $95 \%$ CI & P-value & HR & $95 \% \mathrm{CI}$ & P-value \\
\hline \multicolumn{7}{|l|}{ Sex } \\
\hline Female & 1 & & & 1 & & \\
\hline Male & 1.55 & $1.05-2.28$ & 0.026 & 1.24 & $0.83-1.87$ & 0.3 \\
\hline \multicolumn{7}{|l|}{ Age } \\
\hline$<55$ & 1 & & & 1 & & \\
\hline$\geq 55$ & 1.65 & $1.1-2.48$ & 0.015 & 1.63 & $1.06-2.53$ & 0.028 \\
\hline \multicolumn{7}{|c|}{ Tumor location } \\
\hline Nongastric & 1 & & & 1 & & \\
\hline Gastric & 1.26 & $0.8-2.02$ & 0.32 & 1.35 & $0.81-2.24$ & 0.25 \\
\hline \multicolumn{7}{|l|}{ Tumor size } \\
\hline 1 & 1 & & & 1 & & \\
\hline 2 & 1.3 & $0.84-2.01$ & 0.25 & 1.15 & $0.72-1.83$ & 0.55 \\
\hline Unknown & 1.49 & $0.84-2.64$ & 0.17 & 1.44 & $0.66-3.14$ & 0.36 \\
\hline \multicolumn{7}{|c|}{ Mitotic index } \\
\hline$<5 / \mathrm{HPF}$ & 1 & & & 1 & & \\
\hline$\geq 5 / \mathrm{HPF}$ & 1.26 & $0.74-2.14$ & 0.4 & 1.15 & $0.66-2.0$ & 0.64 \\
\hline Unknown & 1.54 & $0.86-2.77$ & 0.15 & 1.24 & $0.58-2.64$ & 0.58 \\
\hline \multicolumn{7}{|c|}{ Pre-treatment NLR } \\
\hline$\leq 2.4$ & 1 & & & 1 & & \\
\hline$>2.4$ & 1.85 & $1.26-2.72$ & 0.002 & 1.92 & $1.27-2.91$ & 0.002 \\
\hline \multicolumn{7}{|c|}{ Mutational status } \\
\hline KIT 11 & 2.64 & $1.47-4.73$ & 0.001 & 3.39 & $1.73-6.63$ & $<0.001$ \\
\hline Other & 1 & & & 1 & & \\
\hline Unknown & 2.02 & $1.15-3.54$ & 0.014 & 2.29 & $1.19-4.39$ & 0.013 \\
\hline
\end{tabular}

CI, confidence interval; HPF, high-power field; HR, hazard ratio; NLR, neutrophil-to-lymphocyte ratio.

exon 11 or PDGFRA mutations (68 and $57 \%$ vs. 34 and $15 \%$, respectively) (10). Moreover, a retrospective study on samples obtained from patients included in a phase I/II trial with sunitinib also showed that patients with KIT exon 9 mutations had significantly better objective response rates, PFS and OS than patients harboring KIT exon 11 mutations (11). Previously, we found PDGFRA mutations to be an independent negative prognostic factor for PFS and OS (10); however, this observation was not confirmed in the current study, probably because of the small sample size of patients with available genetic data and the low incidence of PDGFRA mutations in the study population. Moreover, these results have some limitations. We only analyzed the mutational status of primary tumors and did not conduct screenings for secondary mutations acquired during imatinib therapy. Although patients carrying secondary mutations in exon 13 or 14 KIT had longer PFS on sunitinib than patients with exon 17 or 18 KIT mutations (11), the analysis of secondary mutations can also be biased due to high heterogeneity and multiple cell clones having different mutational patterns (30).

This study had some limitations, including its retrospective nature, the presentation of a single institution experience and a moderate sample size. Moreover, the relatively long period of the treatment could have led to numerous biases, including changes in side effect management. Nonetheless, our study is the first to illuminate the role of NLR as a prognostic factor in the second line treatment with sunitinib in patients with GIST. A variety of concurrent conditions, including inflammation, infections, and concomitant medication, may influence neutrophil and lymphocyte counts independently from the tumor. The bias associated with active infection is minimalized, because in such conditions treatment is usually delayed; however, a confounding effect of inflammation cannot be ruled out completely. Moreover, the results of this study cannot be easily compared with other analyses of patients with GIST or other solid malignancies due to the high variability of the cutoff levels used for NLR, which ranged from 2.04 (21) through 2.7 (19), 3.0 (17) to even 5 (31). Further investigations, preferable randomized trials with larger cohorts of patients, are required to confirm the prognostic role of NLR in patients with advanced GIST.

Therefore, until now, only primary tumor mutational status and sunitinib-induced hypertension were confirmed as predictive and prognostic factors in patients with GIST treated with sunitinib in the second-line setting (10). In the 
current study, we showed that NLR is an independent prognostic factor for OS in this group of patients. Moreover, we confirmed an earlier observation that the KIT genotype is independently correlated with OS and PFS. The an identification of NLR as independent prognostic factor of GIST may be very useful in clinical practice because, it is an easily measured, reproducible, widely available, and cost-effective marker of systemic inflammation. Blood NLR can serve as a useful parameter in the selection of appropriate and effective treatment for patients with GIST who have progressed on imatinib. We do not currently have reliable biomarkers within this group of patients. Patients with high NLR and poorer prognoses on sunitinib are likely good candidates for clinical trials with new compounds, especially given that agents such as the new tyrosine kinase inhibitors DCC-2618 and avapritinib are planned to be tested in GIST patients after imatinib failure. Moreover, NLR may be taken into account in the trials including immunotherapy; the first trial including a combination of axitinib and avelumab is ongoing.

\section{Acknowledgements}

The preliminary version of this study was presented as a poster during Annual American Society of Clinical Oncology Congress in 2018 in Chicago, June 1st-5th, 2018, abstract number 11531(https://ascopubs.org/doi/abs/10.1200/JCO.2018. 36.15_suppl.11531).

\section{Funding}

No funding was received.

\section{Authors' contributions}

PS, PT, IL, AMC and PR were involved in conceiving the study. PT, IL, AK, EB, HKP, CO, JS and PR were involved in the data acquisition. PS, PT, IL AMC and PR were involved in the data analysis and interpretation. PS, PT, AMC and PR prepared the manuscript. All authors read and approved the final manuscript.

\section{Availability of data and materials}

The datasets used and/or analyzed during the present study are available from the corresponding author on reasonable request.

\section{Ethics approval and consent to participate}

The study has been approved by the local Bio-Ethics Committee (Bio-Ethics Committee at the Maria Sklodowska-Curie Institute-Oncology Center, Warsaw, Poland; approval number KB/9/2011) according to good clinical practice guidelines. Each patient, at the beginning of treatment provided informed consent for use of their data for future studies.

\section{Patient consent for publication}

Not applicable.

\section{Competing interests}

PR has received for lectures from Novartis, Roche, Pfizer, BMS, Eli Lilly and MSD, and served as a member of the advisory board for Novartis, Merck, Amgen, Blueprint Medicine, Roche, BMS, and MSD. IL has received honoraria for lectures from Novartis, Roche, Pfizer, BMS, and MSD. PT has received honoraria and travel grants from Roche, Eli Lilly, Bayer, and Novartis. PS has received travel grants from Roche and Pierre Fabre.

\section{References}

1. Nilsson B, Bümming P, Meis-Kindblom JM, Odén A, Dortok A, Gustavsson B, Sablinska K and Kindblom LG: Gastrointestinal stromal tumors: The incidence, prevalence, clinical course, and prognostication in the preimatinib mesylate era-a population-based study in western Sweden. Cancer 103: 821-829, 2005.

2. Miettinen M and Lasota J: Gastrointestinal stromal tumors: Pathology and prognosis at different sites. Semin Diagn Pathol 23: 70-83, 2006.

3. Hirota S, Isozaki K, Moriyama Y, Hashimoto K, Nishida T, Ishiguro S, Kawano K, Hanada M, Kurata A, Takeda M, et al: Gain-of-function mutations of c-kit in human gastrointestinal stromal tumors. Science 279: 577-580, 1998.

4. Heinrich MC, Corless CL, Duensing A, McGreevey L, Chen CJ, Joseph N, Singer S, Griffith DJ, Haley A, Town A, et al: PDGFRA activating mutations in gastrointestinal stromal tumors. Science 299: 708-710, 2003.

5. Casali PG, Abecassis N, Bauer S, Biagini R, Bielack S, Bonvalot S, Boukovinas I, Bovee JVMG, Brodowicz T, Broto JM, et al: Gastrointestinal stromal tumours: ESMO-EURACAN clinical practice guidelines for diagnosis, treatment and follow-up. Ann Oncol 29: iv68-iv78, 2018.

6. Van Glabbeke M, Verweij J, Casali PG, Le Cesne A, Hohenberger P, Ray-Coquard I, Schlemmer M, van Oosterom AT, Goldstein D, Sciot R, et al: Initial and late resistance to imatinib in advanced gastrointestinal stromal tumors are predicted by different prognostic factors: A european organisation for research and treatment of cancer-italian sarcoma group-australasian gastrointestinal trials group study. J Clin Oncol 23: 5795-5804, 2005.

7. Demetri GD, van Oosterom AT, Garrett CR, Blackstein ME, Shah MH, Verweij J, McArthur G, Judson IR, Heinrich MC, Morgan JA, et al: Efficacy and safety of sunitinib in patients with advanced gastrointestinal stromal tumour after failure of imatinib: A randomised controlled trial. Lancet 368: 1329-1338, 2006.

8. Joensuu H, Wardelmann E, Sihto H, Eriksson M, Sundby Hall K, Reichardt A, Hartmann JT, Pink D, Cameron S, Hohenberger P, et al: Effect of KIT and PDGFRA mutations on survival in patients with gastrointestinal stromal tumors treated with adjuvant imatinib: An exploratory analysis of a randomized clinical trial. JAMA Oncol 3: 602-609, 2017.

9. Farag S, Somaiah N, Choi H, Heeres B, Wang WL, van Boven H, Nederlof P, Benjamin R, van der Graaf W, Grunhagen D, et al: Clinical characteristics and treatment outcome in a large multicentre observational cohort of PDGFRA exon 18 mutated gastrointestinal stromal tumour patients. Eur J Cancer 76: 76-83, 2017.

10. Rutkowski P, Bylina E, Klimczak A, Switaj T, Falkowski S, Kroc J, Lugowska I, Brzeskwiniewicz M, Melerowicz W, Osuch C, et al: The outcome and predictive factors of sunitinib therapy in advanced gastrointestinal stromal tumors (GIST) after imatinib failure-one institution study. BMC Cancer 12: 107, 2012.

11. Heinrich MC, Maki RG, Corless CL, Antonescu CR, Harlow A, Griffith D, Town A, McKinley A, Ou WB, Fletcher JA, et al: Primary and secondary kinase genotypes correlate with the biological and clinical activity of sunitinib in imatinib-resistant gastrointestinal stromal tumor. J Clin Oncol 26: 5352-5359, 2008.

12. Eisenhauer EA, Therasse P, Bogaerts J, Schwartz LH, Sargent D, Ford R, Dancey J, Arbuck S, Gwyther S, Mooney M, et al: New response evaluation criteria in solid tumours: revised RECIST guideline (version 1.1). Eur J Cancer 45: 228-247, 2009.

13. Liu G, Ke LC and Sun SR: Prognostic value of pretreatment neutrophil-to-lymphocyte ratio in patients with soft tissue sarcoma: A meta-analysis. Medicine (Baltimore) 97: e12176, 2018. 
14. Mei Z, Shi L, Wang B, Yang J, Xiao Z, Du P, Wang Q and Yang W: Prognostic role of pretreatment blood neutrophil-to-lymphocyte ratio in advanced cancer survivors: A systematic review and meta-analysis of 66 cohort studies. Cancer Treat Rev 58: 1-13, 2017.

15. Templeton AJ, McNamara MG, Šeruga B, Vera-Badillo FE, Aneja P, Ocaña A, Leibowitz-Amit R, Sonpavde G, Knox JJ, Tran B, et al: Prognostic role of neutrophil-to-lymphocyte ratio in solid tumors: A systematic review and meta-analysis. J Natl Cancer Inst 106: dju124, 2014.

16. Guthrie GJ, Charles KA, Roxburgh CS, Horgan PG, McMillan DC and Clarke SJ: The systemic inflammation-based neutrophil-lymphocyte ratio: Experience in patients with cancer. Crit Rev Oncol Hematol 88: 218-230, 2013.

17. Goh BK, Chok AY, Allen JC Jr, Quek R, Teo MC, Chow PK, Chung AY, Ong HS and Wong WK: Blood neutrophil-tolymphocyte and platelet-to-lymphocyte ratios are independent prognostic factors for surgically resected gastrointestinal stromal tumors. Surgery 159: 1146-1156, 2016.

18. Stotz M, Liegl-Atzwanger B, Posch F, Mrsic E, Thalhammer M, Stojakovic T, Bezan A, Pichler M, Gerger A and Szkandera J: Blood-based biomarkers are associated with disease recurrence and survival in gastrointestinal stroma tumor patients after surgical resection. PLoS One 11: e0159448, 2016.

19. Perez DR, Baser RE, Cavnar MJ, Balachandran VP, Antonescu CR, Tap WD, Strong VE, Brennan MF, Coit DG Singer S and Dematteo RP: Blood neutrophil-to-lymphocyte ratio is prognostic in gastrointestinal stromal tumor. Ann Surg Oncol 20: 593-599, 2013

20. Jiang C, Hu WM, Liao FX, Yang Q, Chen P, Rong YM, Guo GF, Yin CX, Zhang B, He WZ and Xia LP: Elevated preoperative neutrophil-to-lymphocyte ratio is associated with poor prognosis in gastrointestinal stromal tumor patients. OncoTargets Ther 9: 877-883, 2016

21. Racz JM, Cleghorn MC, Jimenez MC, Atenafu EG, Jackson TD, Okrainec A, Venkat Raghavan L and Quereshy FA: Predictive ability of blood neutrophil-to-lymphocyte and platelet-to-lymphocyte ratios in gastrointestinal stromal tumors. Ann Surg Oncol 22: 2343-2350, 2015
22. Hanahan D and Weinberg RA: Hallmarks of cancer: The next generation. Cell 144: 646-674, 2011.

23. Galdiero MR, Bonavita E, Barajon I, Garlanda C, Mantovani A and Jaillon S: Tumor associated macrophages and neutrophils in cancer. Immunobiology 218: 1402-1410, 2013.

24. Petrie HT, Klassen LW and Kay HD: Inhibition of human cytotoxic $\mathrm{T}$ lymphocyte activity in vitro by autologous peripheral blood granulocytes. J Immunol 134: 230-234, 1985.

25. Dumitru CA, Lang S and Brandau S: Modulation of neutrophil granulocytes in the tumor microenvironment: Mechanisms and consequences for tumor progression. Semin Cancer Biol 23: 141-148, 2013.

26. Motomura T, Shirabe K, Mano Y, Muto J, Toshima T, Umemoto Y, Fukuhara T, Uchiyama H, Ikegami T, Yoshizumi T, et al: Neutrophil-lymphocyte ratio reflects hepatocellular carcinoma recurrence after liver transplantation via inflammatory microenvironment. J Hepatol 58: 58-64, 2013.

27. Kantola T, Klintrup K, Väyrynen JP, Vornanen J, Bloigu R, Karhu T, Herzig KH, Näpänkangas J, Mäkelä J, Karttunen TJ, et al: Stage-dependent alterations of the serum cytokine pattern in colorectal carcinoma. Br J Cancer 107: 1729-1736, 2012.

28. Elliott RL and Blobe GC: Role of transforming growth factor Beta in human cancer. J Clin Oncol 23: 2078-2093, 2005.

29. Yang L, Pang Y and Moses HL: TGF-beta and immune cells: An important regulatory axis in the tumor microenvironment and progression. Trends Immunology 31: 220-227, 2010.

30. Wardelmann E, Merkelbach-Bruse S, Pauls K, Thomas N, Schildhaus HU, Heinicke T, Speidel N, Pietsch T, Buettner R, Pink D, et al: Polyclonal evolution of multiple secondary KIT mutations in gastrointestinal stromal tumors under treatment with imatinib mesylate. Clin Cancer Res 12: 1743-1749, 2006.

31. Proctor MJ, Morrison DS, Talwar D, Balmer SM, Fletcher CD, O'Reilly DS, Foulis AK, Horgan PG and McMillan DC: A comparison of inflammation-based prognostic scores in patients with cancer. A glasgow inflammation outcome study. Eur J Cancer 47: 2633-2641, 2011. 